\title{
Extraction of Region with Excessive Disparities Using Block Based Disparity Calculation
}

\author{
Sang Hyun Kim, Jeong Yeop Kim, and Gil Ja So
}

\begin{abstract}
In this paper, we propose the method extracts regions with excessive disparities in 3D stereoscopic images using block based disparity calculation for fatigue evaluation. The proposed method calculates the disparity-map using not pixel based method but block based method. The disparity-map's resolution is decreased in proportion to block size. In the proposed method, first, the disparity-map is calculated based on BMA (block matching algorithm). The normalized cross-correlation is used as the cost function. The excessive disparity regions are extracted using the automatically decided threshold with Otsu algorithm in the disparity-map. Next, to eliminate the small regions, the extracted regions are post processed by morphological filter, erosion and dilation. The sum of the disparities in the object regions which is normalized with image size is used as parameter in fatigue evaluation model. To extract the exact object, the object regions will be segmented in 3D stereoscopic left image using region growing.
\end{abstract}

Index Terms - Block based, Otsu threshold, 3D stereoscopic images, discomfort and fatigue.

\section{INTRODUCTION}

Recently, the demand for 3D stereoscopic images is growing sharply. Typically, the real 3D stereoscopic image contents are made by bilateral approach using two left and right cameras, in other words, each side of the camera to acquire images at the same time. If $3 \mathrm{D}$ stereoscopic images using left and right cameras are inputted left and right eyes. The human brain fuses the left and right images and, from retinal disparity. Because our eyes are horizontally separated, each eye has its own perspective of the world, and thus both eyes receive slightly different. Stereoscopic imaging is the perception of depth that is constructed based on the difference between these two retinal images. This approach should ensure uniformity of left and right images to provide the safe and comfortable viewing [1], [2].

Stereoscopic image viewing comfort is one of the main problems that should be solved before the mass market proliferation of stereoscopic 3D content services. The complaints of some viewers about visual discomfort such as

Manuscript received November 30, 2014; revised May 11, 2015. This work was partially supported by Ministry of Science, ICT and Future Planning (MSIP) and Institute for Information \&communications Technology Promotion (IITP) in the Information Communication Technology (ICT) and Culture Technology (CT) Research \&Development Program 2014

S. H. Kim is with the Department of Computer Engineering of the University of Young-San at Yangsan, Kyungnam, Korea (e-mail: ksh50@ysu.ac.kr).

J. Y. Kim is with the School of Undeclared Majors, University Collage of the University of Young-San at Busan, Korea (e-mail: neocopy@ysu.ac.kr).

G. J. So is with the Department of Cyber \& Police Science of the University of Young-San at Yangsan, Kyungnam, Korea (e-mail: kjso@ysu.ac.kr). eyestrain and associated symptoms including nausea and headaches present a barrier in the popularization of stereoscopic 3D technology. Some visual discomfort may occur due to excessive demand on the accommodation-vergence linkage, that is excessive disparities, fast local and global motion, and various stereoscopic distortions [3].

The conventional stereo matching estimates the relation between corresponding pairs, calculates disparities and depth information. It is the method [4]-[6] to describe 3D surface to acquire depth information for all pixels in the image using interpolation.

The viewer discomfort issue is especially important for children as their vision systems have not grown mature and their inter-pupil distances are shorter than adults. It is need to prevent that the children will not be exposed to excessive disparities and thus have no additional eye fatigue due to $3 \mathrm{D}$ stereoscopic image [7].

In Yuan's method [7], the discomfort and fatigue in 3D stereoscopic images occur from the recognition error of depth information made by excessive positive and negative disparities. These the recognition error of depth information is detected by measurement of perceived depth on screen and fixed by depth tuning methods as the depth shift and the depth scaling. Here, if some perceived depths are outside the pre-defined threshold in histogram of disparity map, those perceived depths are regarded as the excessive disparities and fixed by depth tuning methods.

In detection of the excessive disparities based on the histogram, there are some problems. Because objects in 3D stereoscopic images have three-dimensional position and depth depending on the difference of lighting effects, such as smoking. The excessive disparities are also generated locally by particular objects. The histogram based method dose not correctly detects it because of inaccuracy in disparity detection made from occlusion or noise. The many small isolated excessive disparity regions may be occurred [8]-[10]. To reduce the problem, it is required method eliminate small regions and extract the homogenous regions additionally.

In general, image segmentation methods divide an input image into disjoint sub-regions satisfying homogeneity and connectivity conditions: a region is called homogeneous if all its pixels are homogeneous. A region is called connected if there is a connected path between any two pixels. There are three major approaches to segmentation as follows: binary imaging with threshold, edge-based method, and region-based method [11], [12].

In Kim's method [13], the method to extract objects with excessive disparities in 3D stereoscopic images using cost function considering the pixel intensities and the depth information, disparities. This method can extract the exact object with excessive disparities in pre-defined ROI (region 
of interest). For the correct object extraction, it requires the two prior conditions. They are the correct disparity-map calculation and the extracted object located in ROI fully. The first correct disparity-map calculation is a time-consuming processing. The second object's location occur the instability of the algorithm, because it is not confirmed that objects always located in ROI fully. Thus the fast and robust algorithm is necessary to improve the performance of the object extraction.

In this paper, we propose the method extracts regions with excessive disparities in 3D stereoscopic images using block based disparity calculation for fatigue evaluation. The proposed method calculates the disparity-map using not pixel based method but block based method. The disparity-map's resolution is decreased in proportion to block size. To extract the region with excessive disparities for fatigue evaluation, this approach is possible because it is important to investigate the distribution of the excessive disparity region globally. It is also useful to high speed video processing. In the proposed method, first, the disparity-map is calculated based on BMA (block matching algorithm). The normalized crosscorrelation is used as the cost function. The excessive disparity regions are extracted using the automatically decided threshold with Otsu algorithm in the disparity-map. Next, to eliminate the small regions, the extracted regions are post processed by morphological filter, erosion and dilation. The extracted regions are labeled to object regions and the other regions are labeled to background regions. The sum of the disparities in the object regions which is normalized with image size is used as parameter in fatigue evaluation model. To extract the exact object, the object regions will be segmented in 3D stereoscopic left image using region growing which is mentioned in previous paragraph.

\section{ThE PREVIOUS RESEARCHES}

\section{A. Yuan's Method [7]}

This method proposes the depth tuning method. The method starts by estimating 2D image disparities between the left and right images. The viewer's preference, e.g., input through the TV remote, is also fed into the algorithm. A human visual model is then applied to predict a comfortable range of image disparities.

The comfortable disparity range is compared to the image disparities as well as viewer's preference, leading to the decision of how much depth tuning is needed. Finally a view synthesis algorithm is applied to generate newer versions of the left and right images.

To estimate disparities, a regularized block matching algorithm is used between left and right images. Human visual comfort analysis algorithm computes the comfortable range of disparities. The depth tuning method, namely "depth shifting," applies lateral shifting to the two images. The next depth tuning method, "depth scaling", produces a new disparity map with scaled disparities [14].

In Fig. 2, this method's depth tuned image result from "Monsters vs Aliens 3D". This method automatically detects that the image disparities (Fig. 2(c)) are out of the comfort zone, and applies depth scaling method. In this image, the only big red ball region is located out of the comfort zone. The disparities of the big red ball are shifted and scaled. In this method, generally the discomfort region does not match the specific object directly. To apply depth tuning method, it is necessary not to use the simple histogram threshold algorithm but the effective object extraction method.

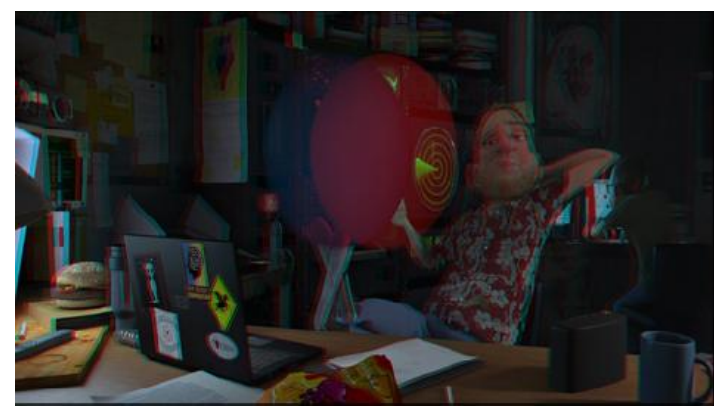

(a)

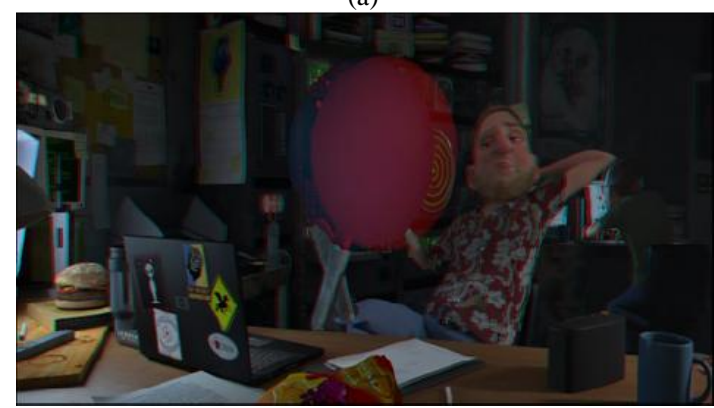

(b)

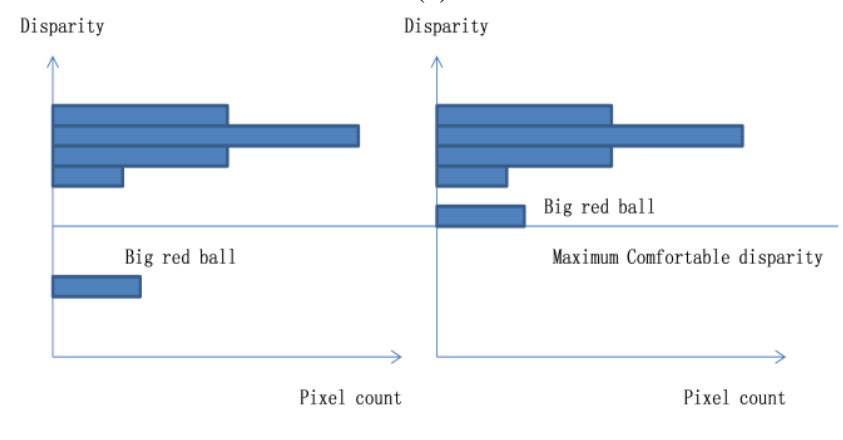

Disparity Histogram of Original image Disparity Histogram of the Tuned Image (c)

Fig. 1. 3D depth tuning and analysis results on the "Monsters vs Aliens 3D" image [7]: (a) Original image pair from "Monsters vs Aliens"; (b) tuned image pair from "Monsters vs Aliens"; (c) visual Comport analysis of the 3D image.

\section{B. Kim's Method [13]}

Kim's method extracts objects with excessive disparities in $3 \mathrm{D}$ stereoscopic images using cost function considering the pixel intensities and the depth information, disparities. The conventional segmentation method considers the only pixel intensities in 2D images. Because 3D stereoscopic images have the additional information that is depth or disparities, the proposed method considers the both pixel intensities and the disparities.

The block diagram of the proposed method is as follows: In Fig. 2, the first the proposed method generates the disparity-map based on left image. The histogram is calculated from the disparity-map. The excessive disparity candidate regions are decided using the pre-defined threshold in the disparity-map. The extracted excessive disparity candidate regions are labeled to object regions and the other regions are labeled to background regions. The regions labeled as object are set as region that will be segmented in 3D stereoscopic left image. The proposed segmentation 
method considering the intensity and the depth information is applied to these regions in 3D stereoscopic left image. Finally to eliminate the protruding regions or the small regions, the morphological filter by means of the post processing is used.

Disparity calculation: A block matching algorithm is used to calculate disparities between left and right images. In our algorithm, we cannot adopt the additive occlusion detection and processing algorithm. To reduce the impression of simulation results come from the disparity calculation error, we used the ground images which are provided by Middlebury dataset by means of disparity-map.

Binarization based on histogram: A pixel in disparity-map which has value is over the pre-defined threshold, classified into the excessive disparity candidate pixel. The threshold can be decided by user preference or recommended based on human visual comfort analysis algorithm [7].

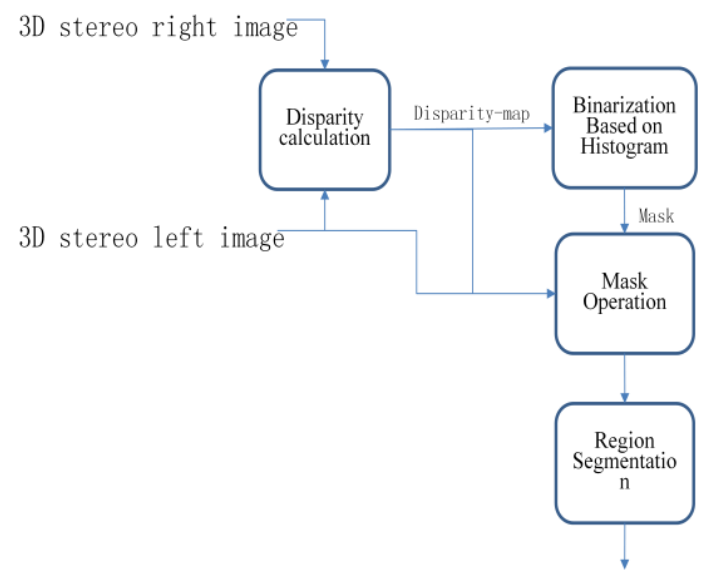

Excessive Disparity Objects Fig. 2. Block diagram of the proposed method.

Mask operation: The extracted excessive disparity candidate pixels are labeled to object pixels and the other pixels are labeled to background pixels. In 3D stereoscopic left image, the overlapped pixels with the pixels which are labeled to object in disparity-map preserve the original pixel value. The other pixels except the overlapped pixels are reset to ' 0 '.

Region Segmentation: The masked 3D stereoscopic left image will be segmented using image segmentation method. The goal of image segmentation is to partition an input image into disjoint sub-regions satisfying homogeneity and connectivity conditions: a region is called homogeneous if all its pixels are homogeneous. A region is called connected if there is a connected path between any two pixels.

Precisely, a segmentation of an image $\mathrm{R}$ is a partition of $\mathrm{R}$ into disjoint sub-regions $R_{1}, R_{2}, \ldots, R_{N}$ satisfying the following conditions:

1) $\bigcup_{i=1}^{N} R_{i}=R$

2) $\quad C\left(R_{i}\right)=$ TRUE for $i=1,2, \ldots, N$ and $C$ : connectivity

3) $H\left(R_{i}\right)=$ TRUE for $i=1,2, \ldots, N$ and $H$ : homogeneity predicate

4) $\quad H\left(R_{i} \cup R_{j}\right)=$ FALSE for any adjacent $R_{i}$ and $R_{j}$

There are three major approaches to segmentation as follows: binary imaging with threshold, edge-based method, and region-based method.

In the various conventional segmentation methods, region-based method is robust and to the image noise better than the others. CLRG (Centroid Linkage Region Growing) [15] is the region-based method which is the simple and the most frequently used. This method's structure is as follows:

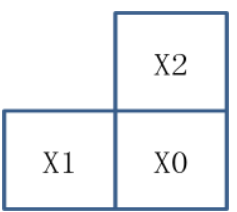

Fig. 3. Region growing geometry of centroid linkage method.

Step 1: Scan the image in the raster scanning order.

Step 2: Compare the pixel $X_{0}$ in scanning to the mean of a neighboring region to which each neighboring pixel $X_{i}, i=1$, 2, belongs.

Step 3: If the pixel $X_{0}$ and the mean of the closest region is close enough, then add the pixel to the region and if the merged region is close enough with another neighboring region then merge the two regions. Else establish a new region having the pixel $X_{0}$. The cost function $C(\cdot)$ is used in comparison with a pixel $X_{0}$ and neighboring region $\mathrm{R}_{\mathrm{m}}$ can be represented as follows:

$$
C\left(R_{m}, X_{0}\right)=\sqrt{\left(\overline{R_{m}}-I\left(X_{0}\right)\right)^{2}}
$$

here, $\overline{R_{m}}$ is the intensity mean of the neighboring $m$-th region $R_{m}$, and $I\left(X_{0}\right)$ is intensity value of a pixel $X_{0}$.

Step 4: Move to the next pixel and then go to step 2.

This segmentation method as CLRG based on the only pixel intensity have problem cannot distinguish the neighboring regions have the similar intensities at region boundary partially. In the entire image the specific target object detection is not easy. Although in our method, the mask operation improve the performance of the target object segmentation to reduce range of segmentation, the additional method is required. 3D stereoscopic images have the additional information that is depth or disparities. We can modify the equation (3) to cost function considering the additional depth information as follows:

$$
C\left(R_{m}, X_{0}\right)=\sqrt{\left(\overline{R_{m}}-I\left(X_{0}\right)\right)^{2}+\alpha\left(\overline{D_{m}}-d\left(X_{0}\right)\right)^{2}}
$$

here, $\overline{D_{m}}$ is the disparity mean of the neighboring $m$-th region $R_{m}$, and $d\left(X_{0}\right)$ is disparity value of a pixel $X_{0}, \alpha$ is the constant.

Fig. 4 shows the segmentation results in the masked 3D stereoscopic left image. Fig. 4(a) is the result of the conventional method using the only intensity. Fig. 4(b) is the result of the Kim's method using the intensity and depth information. In Fig. 4(a), a small plaster manipulation is partitioned to a number of small regions but the front part of table is made to a region linked to small plaster manipulation. It's impossible to separate the small plaster manipulation from the front part of table using the post processing. On the other hand, in Fig. 4(b), the Kim's method result, the front part of table is partitioned to a number of small regions because its disparities are spread. The small plaster manipulation is made to almost a region. The small plaster manipulation can be extracted by using the post processing as morphological filter to eliminate the protruding regions or the 
small regions.

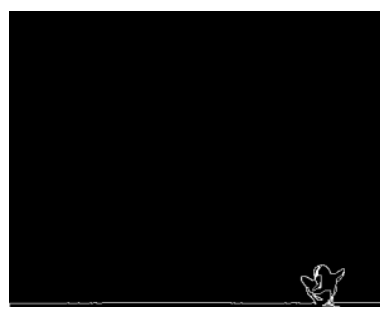

(a)

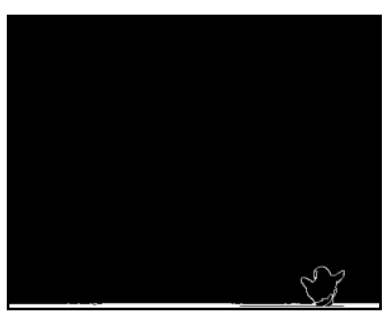

(b)
Fig. 4. Region segmentation results in the masked left image: (a) Region segmentation using intensity in left image; (b) region segmentation using intensity and disparity in left image.

This method can extract the exact object with excessive disparities in pre-defined ROI (region of interest). For the correct object extraction, it requires the two prior conditions. They are the correct disparity-map calculation and the extracted object located in ROI fully. The first correct disparity-map calculation is a time-consuming processing. The second object's location occur the instability of the algorithm, because it is not confirmed that objects always located in ROI fully. Thus the fast and robust algorithm is necessary to improve the performance of the object extraction.

\section{THE PROPOSED METHOD}

We propose the method extracts regions with excessive disparities in 3D stereoscopic images using block based disparity calculation for fatigue evaluation. The proposed method calculates the disparity-map using not pixel based method but block based method. The disparity-map's resolution is decreased in proportion to block size. To extract the region with excessive disparities for fatigue evaluation, this approach is possible because it is important to investigate the distribution of the excessive disparity region globally. It is also useful to high speed video processing.

The block diagram of the proposed method is as follows: In Fig. 5, in the proposed method, first, the disparity-map is calculated based on BMA (block matching algorithm). The normalized cross- correlation is used as the cost function. The excessive disparity regions are extracted using the automatically decided threshold with Otsu algorithm in the disparity-map. Next, to eliminate the small regions, the extracted regions are post processed by morphological filter, erosion and dilation. The extracted regions are labeled to object regions and the other regions are labeled to background regions. The sum of the disparities in the object regions which is normalized with image size is used as parameter in fatigue evaluation model. To extract the exact object, the object regions will be segmented in $3 \mathrm{D}$ stereoscopic left image using region growing which is mentioned in previous paragraph.

Disparity calculation: A block matching algorithm is used to calculate disparities between left and right images. In our algorithm, we cannot adopt the additive occlusion detection and processing algorithm. The normalized cross-correlation is used as the cost function. The normalized cross-correlation equation is as follows: In equation (3), we find the displacement $d_{\max }$ as the disparity value of the block, maximizes the normalized cross-correlation $C(d)$.

$$
C(d)=\frac{\sum_{m \in M} \sum_{n \in N} I_{l}(m, n) I_{r}(m, n-d)}{\sqrt{\sum_{m \in M} \sum_{n \in N} I_{l}(m, n)^{2} \sum_{m \in M} \sum_{n \in N} I_{r}(m, n-d)^{2}}}
$$

here $I_{l}(m, n), I_{r}(m, n-d)$ are left and right images, M, N are height and width of the block. In case of the maximum normalized cross-correlation $C\left(d_{\max }\right)$ is less than the pre-defined value " 0.7 ", we think the occlusion occurs and replaces the disparity value $d_{\max }$ to the previous block's disparity value. We did not compensate the mismatch which is occurred from the structural difference between the left and right images.

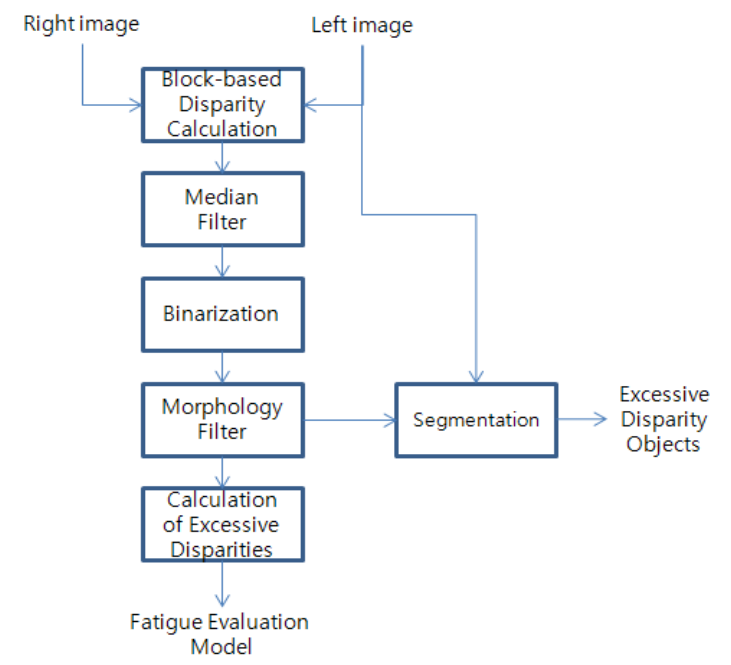

Fig. 5. Block diagram of the proposed method.

Median Filter: In the block based disparity calculation, there are a number of noises as hole which is occurred by mismatching or occlusion. These noises can be removed using median filter. In median filtering, each pixel replaced by the median of the pixels contained in a block around the center pixel. It reduces the impulse noise and preserves edges well.

Adaptive Threshold Calculation and Binarization: The histogram represents the relative frequency of occurrence of the various gray levels in the image. The histogram of disparity-map is generally not uniform but concentrated in the specific range. To decide the adaptive threshold, we first find the upper and lower limits of the range that the histogram is concentrated. In the only upper $75 \%$ area between upper and lower limits, we apply the Otsu's threshold decision method. This method selects the value that maximizes the between-class variance defined as:

$$
\sigma_{B}^{2}(T)=P_{1}(T)\left(\mu_{1}(T)-\mu\right)^{2}+P_{2}(T)\left(\mu_{2}(T)-\mu\right)^{2}
$$

here, $\mu$ the mean of the total pixels is, $\mu_{i}(T)$ is the mean of each class defined by the threshold $T$, and $P_{i}(T)$ is the relative frequency of each class. To determine the threshold $T$ maximizes $\sigma_{B}^{2}(T), \mu_{i}(T)$ and $P_{i}(T)$ have to be determined for all $T$. If the value of a pixel is over the threshold $T$, it is classified into the excessive disparity candidate pixel. Each pixel in disparity-map which has value is over the automatically defined threshold $T$. The extracted excessive disparity candidate pixels are labeled to object pixels and the other pixels are labeled to background pixels. 
Morphology Filter: To eliminate the isolated small regions, morphological filter, erosion and dilation, is applied to binary image. Dilation is the morphological transformation which combines two sets using vector addition of set elements. If $A$ and $B$ are sets in $N$-space $\left(E^{N}\right)$ with elements $a$ and $b$, respectively, $a=\left(a_{1}, \cdots, a_{N}\right)$ and $b=\left(b_{1}, \cdots, b_{N}\right)$ being $\mathrm{N}$-tuples of element coordinates, then the dilation of A by $\mathrm{B}$ is the set of all possible vector sums of pairs of elements, one coming form A and one coming from B [16]. Erosion is the morphological dual to dilation. It is the morphological transformation which combines two sets using the vector subtraction of set elements.

Calculation of Excessive Disparities: In general, the discomfort and fatigue in 3D stereoscopic images is proportional to the excessive positive and negative disparities The discomfort and fatigue equation can be defined as follows:

$$
F(\cdot) \cong S_{d}
$$

here, $F(\cdot)$ is the discomfort and fatigue, $\alpha$ is a proportional constant, and $S_{d}$ is the normalized sum of disparities in the excessive disparity region. To normalize the sum of disparities, the image size is used.

Segmentation: finally in the excessive disparity region, we can extract the object using region segmentation. It have a problem that the user do not know automatically whether the object be fully or partially in the excessive disparity region. Thus as semi-automatic method, the object extraction is processed based on the user's selection. In this paper, we do not implement this part.

\section{Simulation Results}

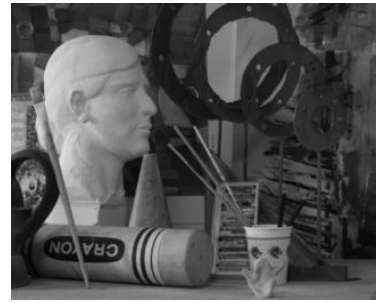

(a)

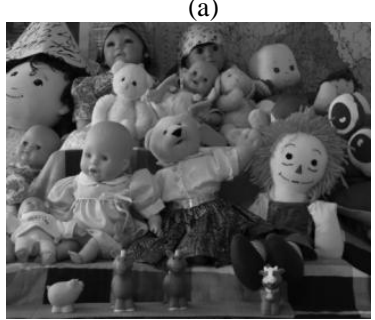

(c)

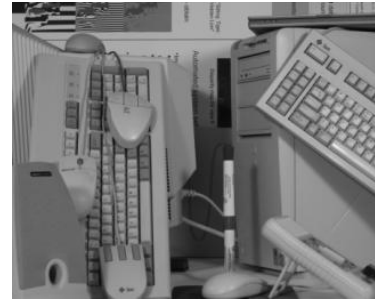

(b)

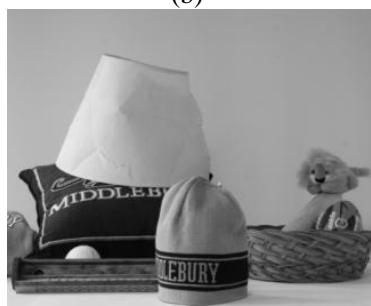

(d)
Fig. 6. The Simulation 3D stereoscopic images: (a) art; (b) computer; (c) dolls; (d) midd2.

The 3D stereoscopic images in Middlebury University dataset [17] were used in this paper's simulation. Fig. 6 shows the simulation original images. In Middlebury University dataset, 3D stereoscopic images are composed of images with the full and half size generated by 3 types of lighting and exposure. The original 3D stereoscopic images are color images. It is converted into gray image. Fig. 7 shows the disparity-maps, ground images in Middlebury
University dataset. In Fig. 7, the more pixel intensity is bright, the more its disparity value is strong. For example, a small plaster manipulation and the front part of table in Fig. 7(a) are the brightest and highest disparities in this disparitymap. Fig. 8 shows the proposed disparity calculation results. The disparity-map's resolution is decreased in proportion to block size. Here the block size is the $8 \times 8$. The image resolution decreased to $1 / 64$. To extract the region with excessive disparities for fatigue evaluation, this approach is possible because it is important to investigate the distribution of the excessive disparity region globally. In Fig. 8, the horizontal streak is occurred in case of the maximum normalized cross-correlation is less than the pre-defined value. In that case, we replace the disparity value to the previous block's disparity value. For visualization, it is two times the disparity values.

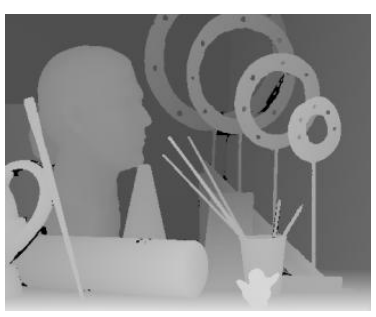

(a)

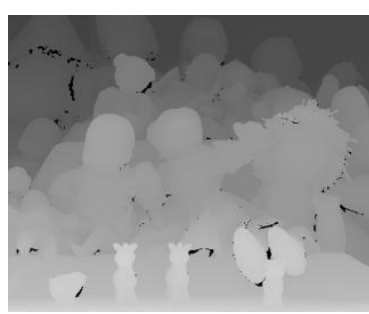

(c)

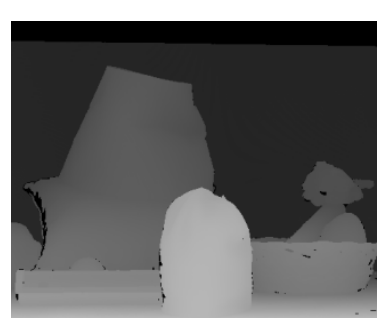

(d)

Fig. 7. Disparity-map of Art image: (a) art; (b) dolls; (c) midd2.

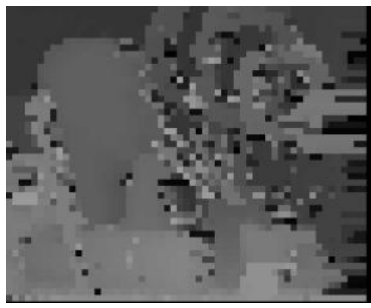

(a)

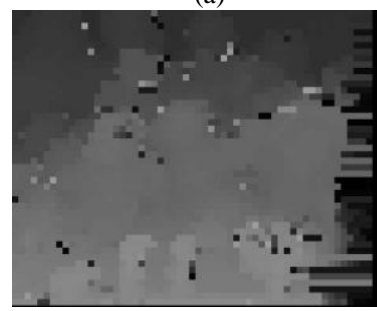

(c)

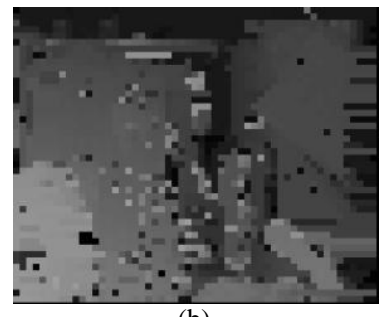

(b)

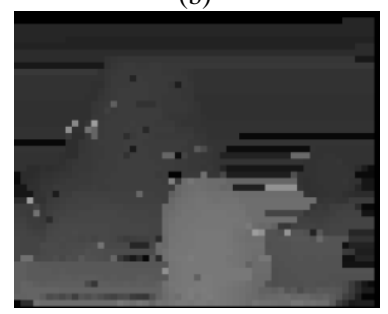

(d)
Fig. 8. The proposed disparity calculation results: (a) art; (b) computer; (c) dolls; (d) midd2.

Fig. 9 shows the median filtering results. The median filter replaces each pixel to the median of the pixels contained in a block around the center pixel. The boundary of the image is not processed. Except the boundary of the image, the median filter reduces the impulse noise and preserves edges well. Fig. 10 shows the excessive disparity regions are extracted using the automatically decided threshold with Otsu algorithm in the disparity-map. In Fig. 10, we can see that the highlight regions at the bottom of each image are extracted as the 
excessive disparity regions. Fig. 11 shows the elimination of the isolated small regions using the morphological filter, opening be made of erosion and dilation. In Fig. 11, the isolated white object small regions and black hole small regions are eliminated by the morphological filter. Fig. 12 shows the overlapped parts in 3D stereoscopic left image with the excessive disparity regions. In Fig. 12, we can see that the protruding objects in the original images are located in the extracted excessive disparity regions. Table I shows that the threshold $\mathrm{T}$ decided by Otsu algorithm, the total number of pixels in the excessive disparity regions, and the normalized sum of disparities $S_{d}$ which is used as parameter in the discomfort and fatigue equation (5) in the four simulation 3D stereoscopic images.

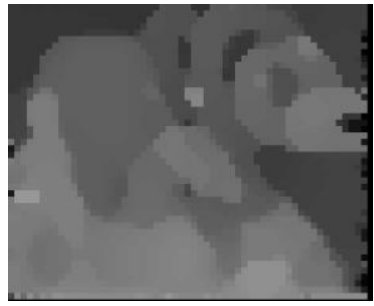

(a)

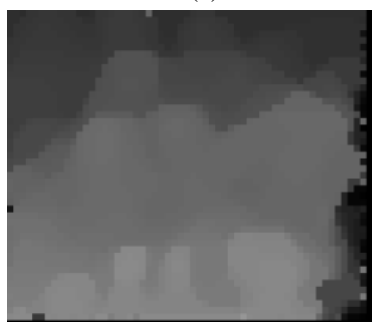

(c)

Fig. 9. The median filtering results: (a) art; (b) computer; (c) dolls; (d) midd2.
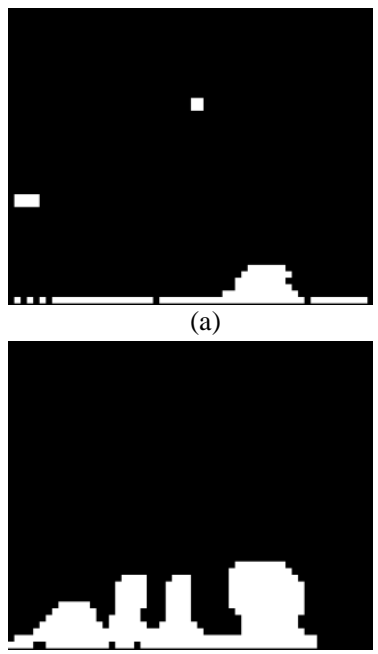

(c)

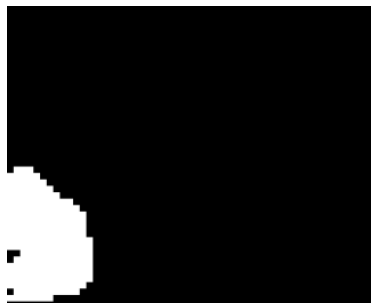

(b)

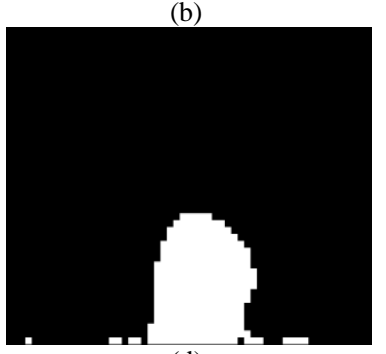

(d)
Fig. 10. The excessive disparity regions are extracted using the automatically decided threshold with Otsu algorithm: (a) art; (b) computer; (c) dolls; (d) $\operatorname{midd} 2$.

TABLE I: THE PARAMETERS FOR THE DISCOMFORT AND FATIGUE EQUATION

\begin{tabular}{cccc}
\multicolumn{4}{c}{ EQUATION } \\
\hline \hline Image Name & $\begin{array}{l}\text { Threshold } \\
(T)\end{array}$ & $\begin{array}{l}\text { Number of Pixels } \\
\text { in Excessive } \\
\text { Disparity Region }\end{array}$ & $\begin{array}{l}\text { Normalized Sum } \\
\text { of Disparities }\left(S_{d}\right)\end{array}$ \\
\hline Art & 134 & 4464 & 3.45 \\
Computer & 140 & 15376 & 13.24 \\
Dolls & 130 & 26496 & 19.80 \\
Midd2 & 112 & 18384 & 12.56 \\
\hline \hline
\end{tabular}

In Table I, the image "Midd2" has the more number of pixels in the excessive disparity regions than the number of pixels in the image "Computer". But the normalized sum of disparities of the image "Computer" is greater than the normalized sum of disparities in the image "Midd2", because the threshold in image "Computer" is higher than the threshold in the image "Midd2".
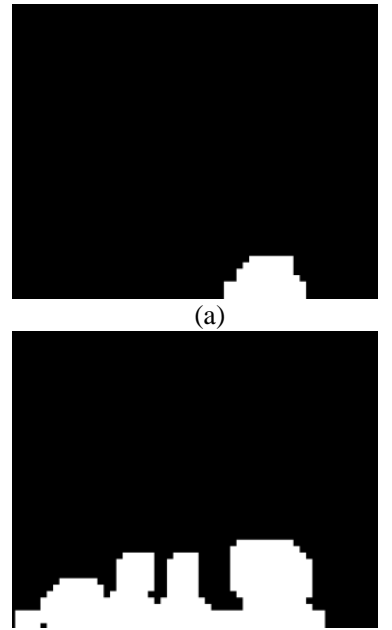

(c)

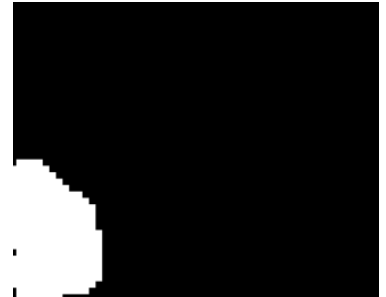

(b)

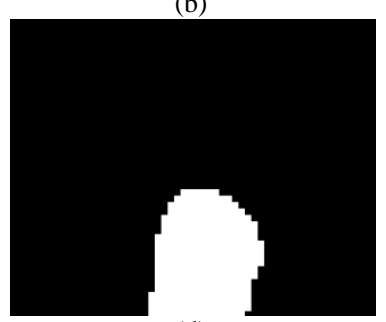

(d)
Fig. 11. The elimination of the isolated small regions using the morphological filter: (a) art; (b) computer; (c) dolls; (d) midd2.

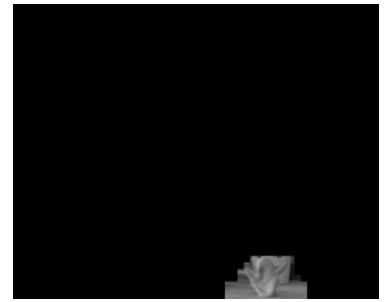

(a)

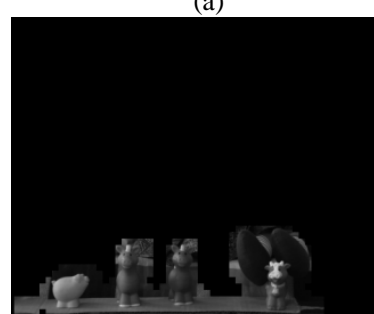

(c)

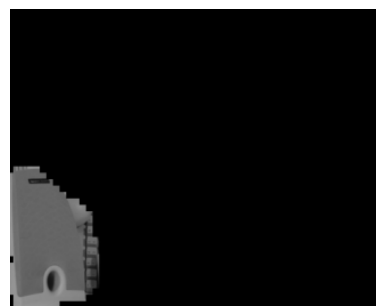

(b)

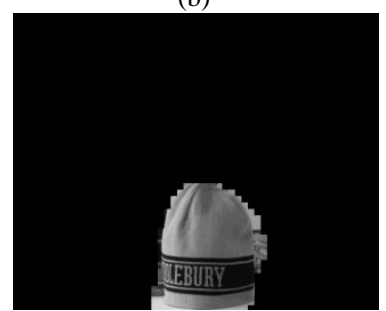

(d)
Fig. 12. The overlapped parts in 3D stereoscopic left image with the excessive disparity regions: (a) art; (b) computer; (c) dolls; (d) midd2.

\section{CONCLUSION}

Stereoscopic image viewing comfort is one of the main problems that should be solved before the mass market proliferation of stereoscopic 3D content services. The discomfort and fatigue in 3D stereoscopic images occur from the recognition error of depth information made by excessive positive and negative disparities.

In this paper, we propose the method extracts regions with excessive disparities in 3D stereoscopic images using block based disparity calculation for fatigue evaluation. The proposed method calculates the disparity-map using not pixel based method but block based method. The disparity-map's resolution is decreased in proportion to block size. To extract the region with excessive disparities for fatigue evaluation, this approach is possible because it is important to investigate the distribution of the excessive disparity region globally. It 
is also useful to high speed video processing. In the proposed method, first, the disparity-map is calculated based on BMA. The normalized cross- correlation is used as the cost function. The excessive disparity regions are extracted using the automatically decided threshold with Otsu algorithm in the disparity-map. Next, to eliminate the small regions, the extracted regions are post processed by morphological filter, erosion and dilation. The extracted regions are labeled to object regions and the other regions are labeled to background regions. The sum of the disparities in the object regions which is normalized with image size is used as parameter in fatigue evaluation model. To extract the exact object, the object regions are segmented in 3D stereoscopic left image using region growing.

We did not implement the object extraction using region segmentation or growing because it have a problem that the user do not know automatically whether the object be fully or partially in the excessive disparity region. In future, it is necessary to study the semi-automatic method based on the user's selection.

\section{ACKNOWLEDGMENT}

This research is partially supported by Ministry of Science, ICT and Future Planning (MSIP) and Institute for Information \&communications Technology Promotion (IITP) in the Information Communication Technology (ICT) and Culture Technology (CT) Research \&Development Program 2014.

\section{REFERENCES}

[1] C. W. Choi, " HUD factor classification of online FPS Game for 3D stereoscopic application," M.S. thesis, Dept. of Media Broadcasting, Graduate School of Information Sciences, Soongsil Univ., Seoul, Korea, 2011.

[2] S. Choi, "Technical status and prospect of 3D stereoscopic images," Journal of Korea Information Processing, vol. 17, no. 4, pp. 4-11, 2010.

[3] M. Lambooji, W. A. IJsselsteijn, and I. Heynderickx, "Visual discomfort of 3D TV: Assessment methods and modeling," Displays, vol. 32, issue 4, Oct. 2011.

[4] J. S. Kim, "A study on a balance and registration of non-rectified stereo images," Ph.D. Dissertation, Chung-Ang Univ., Seoul, Korea, 2009.

[5] S. Kim, G. So, and J. Kim, "Intensity correction of 3D stereoscopic images using region segmentation," in Proc. 34th Conf. Korea Information Processing Society, Seoul, 2010.

[6] J. S. Lee, S. D. Park, S. H. Han, and C. Y. Kim, "Implementation of 3-dimension stereoscope using 3D graphics," in Proc. Conf. Korea Multimedia Society, 2010, vol. 13, no. 2, pp. 184-187.

[7] C. Yuan, H. Pan, and S. Daly, "61.3: Stereoscopic 3D content depth tuning guided by human visual models," in Proc. SID Symposium Digest of Technical Papers, June 2011, vol. 42, issue 1, pp. 916-919.

[8] Y. J. Zhang, "Improving the accuracy of direct histogram specification," Electronics Letters, vol. 28, issue 3, pp. 213-214, 1992.

[9] H. Tuo, L. Zhang, and Y. Liu, "Multisensory aerial image registration using direct histogram specification," in Proc. IEEE Int'l Conf. Sensing and Control, 2004, pp. 807-812, vol. 2.

[10] A. Mancini, "Disparity estimation and intermediate view reconstruction for noble application in stereoscopic video," M.S. thesis, McGill University, 1998.

[11] R. C. Gonzalez and R. E. Woods, Digital Image Processing, 2nd ed., MA: Addison-Wesley, 1992.

[12] A. K. Jain, Fundamental of Digital Image Processing, Printice-Hall, NJ: Englewood Cliffs, 1989.

[13] S. H. Kim, J. Y. Kim, and G. J. So, "Object extraction with excessive disparities in 3D stereoscopic images," International Journal of
Computer Theory and Engineering, vol. 6, no. 4, pp. 313-318, Aug. 2014.

[14] H. Pan, C. Yuan, and S. Daly, "3D video disparity scaling for preference and prevention of discomfort," in Proc. SPIE 7863, Stereoscopic Displays and Applications XXII, Feb. 2011.

[15] R. M. Haralick and L.G. Shapiro, "Image segmentation techniques," Computer Vision, Graphic and Image Processing 29, pp. 100-132, 1985.

[16] R. M. Haralick, S. R. Sternberg, and X. Zhuang, "Image analysis using mathematical morphology," IEEE Trans. on PAMI., vol. 9, pp. 532-550, July 1987.

[17] Middlebury. [Online]. Available: http://vision.middlebury.edu/stereo/

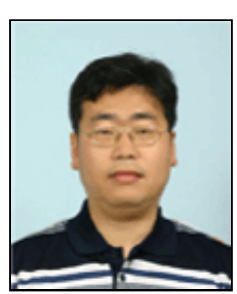

Sang-Hyun Kim received the diploma in electronics engineering from Kyung-Pook National University of Daegu, South Korea in 1991; M.S. and PhD degrees both in image processing of electronics engineering, from the same university, in 1993 and 1998, respectively.

He was with the Samsung Medison Co. Ltd, in Korea as a senior researcher from 1998 to 2001. From September 2001, he is with the Department of Computer Engineering of the University of Young-San at Yangsan, Kyungnam as an associate professor. His doctoral dissertation work focused on the development of fractal image coding method and transmission for image processing, image communication and computer vision problems. The topics he has worked on include virtual reality, ultrasound image processing, medical image processing, image feature extraction and classification, and 3D stereoscopic images. His current research is on region-based intensity correction in 3D stereoscopic images.

$\mathrm{He}$ is a member of the KMMS (Korea Multimedia Society) and KIPS (Korea Information Processing Society).

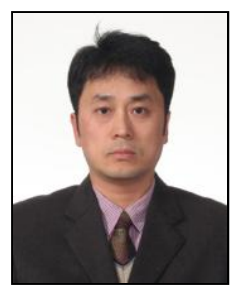

Jeong-Yeop Kim received the diploma in electronics engineering from Kyung-Pook National University of Daegu, South Korea in 1990; M.S. and PhD degrees both in image processing of electronics engineering, from the same university, in 1992 and 2000, respectively.

He was with the Samsung Advanced Institute of Technology in Korea as a senior research staff from 1994 to 2000. From March 2001 to February 2013, he was with the Department of Game Contents Science of the University of Young-San at Busan, as an associate professor. From March 2013, He is with the Department of Undeclared Major of the University of Young-San at Busan. His doctoral dissertation work focused on detecting and adjusting the white point from single color image. The topics he has worked on include color printing, error diffusion, color correction and 3D stereoscopic images. His current research is on correction of color data in 3D stereoscopic images.

$\mathrm{He}$ is a member of the KMMS (Korea Multimedia Society) and KIPS (Korea Information Processing Society).

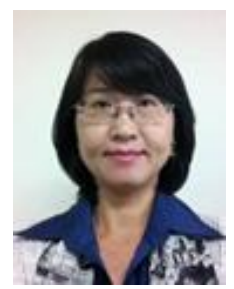

Gil-Ja So received the diploma in computer science from the Dong-Eui University of Pusan, South Korea in 1994; and M.S. and PhD degrees both in natural language processing of computer engineering, from the Pusan National university, South Korea in 1997 and 2012, respectively. From September 2001, she is with the Department of Computer Engineering of the University of Young-San at Yangsan, Kyungnam as an lecturer. Her doctoral dissertation work focused on the Korean grammar checker for parsing Korean documents and correcting context-sensitive words in documents. The topics she has worked on include virtual reality, game development and artificial intelligence of non-players of game, and 3D stereoscopic images. Her current research is on region-based intensity correction in 3D stereoscopic images.

She is a member of the KMMS (Korea Multimedia Society) and KIPS (Korea Information Processing Society). 